\title{
State dependent pseudoresonances and excess noise
}

\author{
Francesco Papoff ${ }^{1}$, Giampaolo D'Alessandro ${ }^{2}$ and G.-L. Oppo ${ }^{3}$ \\ 1 Department of Physics, University of Strathclyde, 107 Rottenrow, Glasgow G4 0NG, UK \\ 2 School of Mathematics, University of Southampton, Southampton SO17 1BJ, England, UK
}

(Dated: March 31, 2008)

\begin{abstract}
We show that strong response to non-resonant modulations and excess noise are state dependent in generic nonlinear systems, i.e. they affect some output states, but are absent from others. This is demonstrated in complex Swift-Hohenberg models relevant to optics, where it is caused by the non-normality of the linearized stability operators around selected output states, even though the cavity modes are orthogonal. In particular, we find the effective parameters that control excess noise and the response to modulations and show cases where these phenomena are enhanced by an order of magnitude.
\end{abstract}

PACS numbers: 42.65.Sf,42.60.Mi,05.45.-a,42.55.Ah

Excess noise [1] is a term used in optical device physics to highlight the fact that the sum of the energy in the individual modes is greater than the total energy available. This phenomenon was first predicted by Petermann in [2] and its paradoxical result was explained in [3]: the modes of excess noise systems are correlated (non-orthogonal). Therefore, the total energy cannot be written as the sum of the energies of the individual modes since one must include the mode-mode correlation terms too. Usually, the "modes" referred to in the explanation of this phenomenon have been interpreted as modes of the optical cavity, either open [4] or unstable [5] or with misaligned elements [6]. However, the "modes" in question are far more general: they are the modes of the dynamics of the system [7]. In this respect excess noise is just one aspect of the enhanced response to modulation and transient growth typical of non-normal operators, i.e. operators that do not commute with their adjoint $[8,9]$. The main feature of non-normal operators is that their eigenvectors are not orthogonal. This can have dramatic consequences in terms of the dynamics of the system: perturbations of stable states can be substantially amplified before they eventually decay (transient growth) [8]; the response to an external modulation can be very large even far from resonance (pseudo-resonance) [10]. An essential point often overlooked is that when the system under investigation is nonlinear, then the operator responsible for these effects is the linear stability operator and this is state, not just model, dependent. This is particularly important when studying systems that are both timeand space-dependent and have a rich bifurcation structure. Enhanced response to external modulations critically affects, for example, the implementation of chaos synchronization in secure laser communications [11].

In this letter we analyze three aspects of generic nonlinear systems. First of all primary bifurcation of a given state may be normal, while its secondary bifurcations, in general, are not. Secondly, the effects of non-normality on the nonlinear dynamics and response can vary from state to state and need to be quantified. Thirdly, the system response depends on the spatial and temporal frequency of the modulation. This opens the possibility of fine tuning the output of the system by carefully choosing the modulation wave number(s) and frequency(ies). We illustrate these aspects here using a Swift-Hohenberg model of lasers [12-14]. By fitting the model parameters to experimental curves, the model can be used to provide qualitatively accurate predictions of the dynamics of a wide variety of lasers near the lasing threshold. Our analysis aims to determine the parameters that have the largest effects on non-normality and, consequently, on modulational response. Swift-Hohenberg equations are a paradigm of weakly nonlinear analysis. Therefore, state dependent excess noise and enhanced modulation response discussed here are not limited to lasers or optics, but apply to any nonlinear system near transitions from stationary states to traveling waves.

The dynamical consequences of non-normality can be understood in terms of simple systems of linear differential equations $[10,15]$. For example, the solution $\boldsymbol{u}(t)=$ $\exp (t A) \boldsymbol{u}(0)$ of $\dot{\boldsymbol{u}}=A \boldsymbol{u}$, with $A$ a non-normal matrix with eigenvalues that have strictly negative real-part, can show transient growth: in other words, $\|\exp (t A) \boldsymbol{u}(0)\|$ may not be a monotonic decreasing function of time, even though $\Re(\lambda)<0$ for all the eigenvalues $\lambda$ of $A$. From here on $\|\boldsymbol{u}\|=\overline{\boldsymbol{u}} \cdot \boldsymbol{u}$ is the norm induced by the scalar product and the overbar ${ }^{-}$symbol indicates complex conjugate. As a second example we note that the amplitude of the asymptotic solution of $\boldsymbol{u}=A \boldsymbol{u}+\boldsymbol{v} \exp \left(i \omega_{0} t\right)$ is not determined by the distance between $\omega_{0}$ and the eigenvalues (spectrum) of $A$, but is proportional to the norm of the resolvent of $A$ evaluated at the point in the complex plane $z=i \omega,\|R(A, i \omega)\| \equiv\left\|(i \omega-A)^{-1}\right\|$. For any operator, the norm we use here is defined as the largest singular value of the operator. If $A$ is nonnormal, this norm may be large, even though the frequency may not be near any of the natural frequencies of the system (pseudo-resonance) [10]. These results can be extended to systems of partial differential equations and have had numerous applications in math- 
ematics and physics [16] from hydrodynamics [10] and geophysics [8, 9] to lasers[4, 7].

To illustrate the state-dependence of non-normality, we consider a generic system of nonlinear partial differential equations

$$
\frac{\partial \boldsymbol{u}}{\partial t}=\mathcal{L}_{0} \boldsymbol{u}(x, t)+\mathcal{N}(\boldsymbol{u}(x, t)),
$$

with $\mathcal{N}$ a nonlinear operator such that $\mathcal{N}(0)=0$. The linear stability operator of a time-independent solution $\boldsymbol{u}_{0}(x)$ of Eq. (1) is $\mathcal{L}_{\boldsymbol{u}_{0}}=\mathcal{L}_{0}+\delta \mathcal{N}_{\boldsymbol{u}_{0}}$, with $\delta \mathcal{N}_{\boldsymbol{u}_{0}}$ being the linearization of $\mathcal{N}$ around $\boldsymbol{u}_{0}$ and $\delta \mathcal{N}_{\boldsymbol{u}_{0}}=0$ for $\boldsymbol{u}_{0}=0$. The presence of the term $\delta \mathcal{N}_{\boldsymbol{u}_{0}}$ means that the normality of $\mathcal{L}_{0}$ does not imply that of $\mathcal{L}_{\boldsymbol{u}_{0}}$ for $\boldsymbol{u}_{0}(x) \neq 0$. Physically, this means that effects such as transient growth and enhanced response to external forcing can be present near states with $\boldsymbol{u}_{0} \neq 0$ even if they are absent near $\boldsymbol{u}_{0}=0$.

We consider now the Swift-Hohenberg model of wide aperture lasers with plane mirrors $[13,14]$

$$
\begin{aligned}
\partial_{t} \tilde{\psi}= & (1+\sigma)^{-1}\left[\sigma(\rho-i \eta)+i a \nabla^{2}-\right. \\
& \left.\frac{\sigma}{(1+\sigma)^{2}}\left(\eta+a \nabla^{2}\right)^{2}-\sigma(1+i \alpha) n\right] \tilde{\psi} \\
\partial_{t} n= & \left(-b+c \nabla^{2}\right) n+|\tilde{\psi}|^{2}
\end{aligned}
$$

with $n$ the population inversion and $\tilde{\psi}$ the field appropriately rescaled. Note that the cavity modes are transverse traveling waves that are mutually orthogonal. The strength of pumping is represented by the parameter $\rho$, while $\eta$ is a scaled atom-cavity detuning. The parameters $a, b, c$ and $\sigma$ are all related to geometrical or material properties of the laser, $b$ is the ratio of the decay times of population inversion and of polarization. The parameter $\alpha$ is the line-enhancement factor: it is proportional to the ratio of the derivatives of the refractive index and of the gain with respect to the population and is different from zero if the gain line is asymmetric. The $\alpha$ factor is positive in bulk semiconductor lasers and quantum well lasers; it can be negative in quantum dot lasers [17, 18] and in Raman lasers [19]. Here we consider for illustration purposes the values $a=0.01, b=0.8, c=0$ and $\sigma=0.1$ that are used in [12]. The results are valid for generic parameter values such as, for example, $b>>1$ and $c$ negligible where a single complex Swift-Hohenberg equation used in the description of pattern formation in a wide variety of scientific disciplines [20] is recovered.

Equations (2) have a trivial solution $(\tilde{\psi}, n)=(0,0)$. As the pump parameter $\rho$ is increased, this solution loses its stability to a time- and space-varying solution,

$$
\tilde{\psi}=A e^{i\left(k_{0} x-\omega_{0} t\right)}, \quad n=n_{0},
$$

with $k_{0}, \omega_{0}$ and $n_{0}$ functions of the laser parameters and $A=b\left[\rho-\left(\eta-a k_{0}^{2}\right)^{2} /(1+\sigma)^{2}\right][14,19]$. The linear stability operator of the trivial solution is normal for all value of $\alpha$. We focus here on the instability of the lasing solution (4) which, instead, is non-normal. The first step in this analysis consists in changing to a moving reference frame by introducing a new field $\psi=\tilde{\psi} \exp -i\left(k_{0} x-\omega_{0} t\right)$. The stability of $\tilde{\psi}$ is determined by a non-autonomous system; that of $\psi$ by an autonomous system to which we can apply the theory summarized earlier.

The linearization of (2) in the traveling reference frame of $\psi$ gives rise to a system of linear coupled partial differential equations, $\boldsymbol{v}=\mathcal{L} \boldsymbol{v}$ in $\boldsymbol{v}=(\phi, \bar{\phi}, \nu)$, where $\phi$ and $\nu$ are the modulations of $\psi$ and $n$ respectively. These equations are block diagonal in Fourier space: each block is a set of three coupled linear equations $\dot{\boldsymbol{v}}_{k}=L_{k} \boldsymbol{v}_{k}$, with $\boldsymbol{v}_{k}=\left(\phi_{k}, \bar{\phi}_{-k}, d_{k}\right)$ the $k$-th Fourier components of $\phi$ and $\sqrt{\sigma /(1+\sigma)} \nu$. $L_{k}$ is given by

$$
L_{k}=\left(\begin{array}{ccc}
L_{+} & 0 & -(1+i \alpha) L_{3} \\
0 & L_{-} & -(1-i \alpha) L_{3} \\
L_{3} & L_{3} & L_{4}
\end{array}\right)
$$

with

$$
\begin{aligned}
& L_{ \pm}=-\frac{i a K_{ \pm}}{1+\sigma}-\frac{\sigma}{(1+\sigma)^{3}}\left(\mp \Delta \eta a K_{ \pm}+a^{2} K_{ \pm}^{2}\right), \\
& L_{3}=\sqrt{\sigma /(1+\sigma)} A, \quad L_{4}=-b-c k^{2} .
\end{aligned}
$$

Here $\Delta \eta=\left(\eta-a k_{0}^{2}\right)$ and $K_{ \pm}=2 k_{0} k \pm k^{2}$.

The equations for $\boldsymbol{v}$ and their block-diagonal representation (5) for $\boldsymbol{v}_{k}$ are of the general form described above. It is straightforward to verify that the matrices $L_{k}$ and, hence, the operator $\mathcal{L}$, are non-normal when $L_{3} \neq 0$. Since $L_{3}$ affects all off-diagonal terms of Eq. (5) and depends linearly on the amplitude $A$ of the lasing state, the effect of non-normality on the dynamics increases with $A$ (i.e. with $b$ ) and with $\alpha$. Therefore, phenomena such as transient growth, excess noise and enhanced response to forcing, which are due to non-normality of $\mathcal{L}$, can appear when the field amplitude of the laser state is non null, even though all cavity modes are orthogonal.

As a first illustration of these phenomena we consider the linear response of the lasing solution to external modulations. This is given by

$$
\frac{\partial \boldsymbol{v}}{\partial t}=\mathcal{L}_{\boldsymbol{u}_{0}} \boldsymbol{v}(x, t)+\boldsymbol{f}(x) e^{z t}
$$

where $\boldsymbol{f}(x) \exp (z t)$, with $z \in \mathbb{C}$, is a time dependent forcing term. Eq. (6) in Fourier space provides the response for each Fourier component $\boldsymbol{v}_{k}$ via the block-diagonal representation of $\mathcal{L}$ given in (5):

$$
\boldsymbol{v}_{k}(t)=e^{L_{k} t} \boldsymbol{v}_{k}(0)+R(z, k) \boldsymbol{f}_{k} e^{z t},
$$

where $R(z, k)$ is the resolvent of $L_{k}, \boldsymbol{v}_{k}(0)$ and $\boldsymbol{f}_{k}$ are the $k$-th component of the initial perturbation and of the modulation, respectively. Physically, the latter correspond to injection of field and carrier density with spatial wave number $k$ into the laser. Solutions in real space can be found by summing over all $k$ 's. When the lasing 


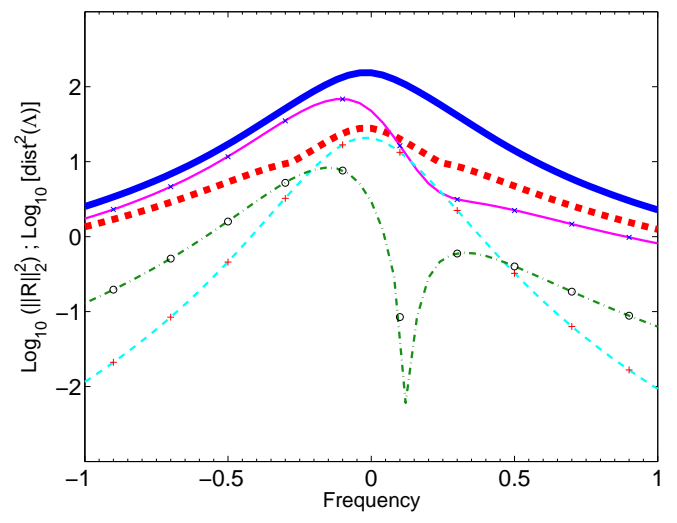

FIG. 1: Response of (2-3) to a monochromatic modulation $\exp \left[i\left(k_{f} x+\omega_{f} t\right)\right]$ of the field $\tilde{\psi}$ as a function of $\omega_{f}$ at $k_{f}=$ 4. The lines are: maximum response of the linear system based on the norm of the resolvent (thick solid blue line) or on the distance from the eigenvalues (thick dashed red line). The other lines represent the expected response of $\phi_{k}$ (solid, magenta), $\phi_{-k}$ (dashed, cyan) and of $\nu_{k}$ (dot-dashed, green). The crosses, pluses and circles are the response measured from direct integration of (2-3) with added forcing term. Parameter values: $\alpha=0.2, \rho=1.5, \eta=-0.09, k_{f}=4$.

solution is stable, the exponential term vanishes after a transient and the maximal response is given by the norm of the resolvent. In particular, for a periodic forcing with $z=i \omega_{f}, \omega_{f} \in \mathbb{R}$, the maximum response amplitude is given by $\left\|\boldsymbol{v}_{k}(t)\right\| \leq\left\|R\left(i \omega_{f}, k\right)\right\|\left\|\boldsymbol{f}_{k}\right\|$.

This has two important consequences. First, for a normal operator $L_{k}$ the optimal response is given by the distance of the forcing frequency $\omega$ to the closest eigenvalues of $L_{k}$. This is very different when $L_{k}$ is nonnormal: quantitatively the maximal response could be much larger than the maximal response of a normal system; qualitatively non-normality can drastically change the frequency ranges corresponding to signal amplification or damping. An example of this behavior is shown in Figure 1, where using the singular value decomposition of the resolvent [8] we show that the maximal response (thick blue solid line) is an order of magnitude larger that the maximal response for a normal systems with the same resonances as the one considered (thick red dashed line).

However, the maximal response is, in general, generated by vectors with components corresponding to different physical quantities, such as the field amplitude and population inversion in the laser case. In practice, one may by more interested in finding the response to monochromatic forcing of either the field or the population inversion, which are more common in experiments. The resolvent provides also the response to these type of forcing. We have added a field forcing term proportional to $\exp \left[i\left(k_{f} x+\omega_{f} t\right)\right]$ to $(2)$. We have measured the amplitude of the modulation of the (stable) traveling wave solution either by direct integration of equations (2-3)

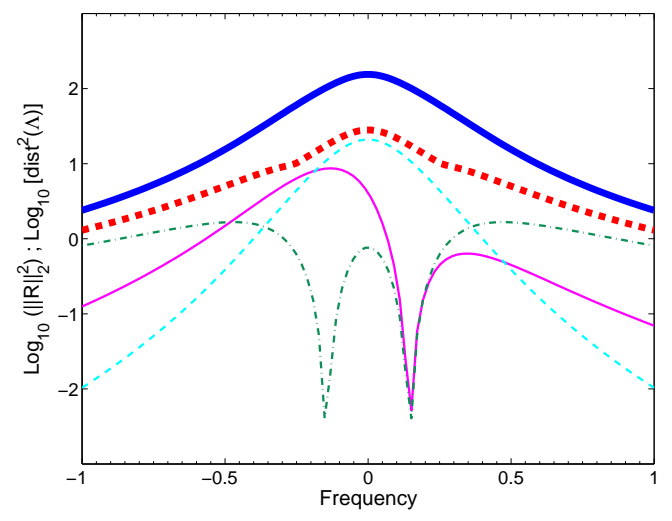

FIG. 2: Response off (2-3) to a modulation $\cos \left(k_{f} x+\omega_{f} t\right)$ of the population inversion $n$ as a function of $\omega_{f}$ at $k_{f}=4$. The lines are as in Figure 1. Parameter values: $\alpha=0.2, \rho=1.5$, $\eta=-0.09, k_{f}=4$.

with added forcing term, or using equation (7). In the particular case of the traveling wave solution (4), the temporal and spatial frequency of the wave and of the modulation are mixed in the laboratory frame. Therefore, the response of the system is determined not just by $R\left(i \omega_{f}, k_{f}\right)$, but also by $R\left[ \pm i \omega_{f}+\omega_{0}, \pm\left(k_{f}-k_{0}\right)\right]$.

Figure 1 shows also the amplitude of the components of $\boldsymbol{v}_{k}$ as a function of the forcing frequency $\omega_{f}$ in the case of single-wavelength forcing at $k_{f}=4$ of the field. The amplitude of the modulation of $\phi_{k}$ is larger than what would have been expected by the distance of $\omega_{f}$ from the eigenvalues of $L_{k}$. Figure 2, instead, shows the response to a monochromatic modulation on the population inversion. In this case a term proportional to $\cos \left(k_{f} x+\omega_{f} t\right)$ was added to (3). In this example, the field modulation is more effective than that of the population inversion: as can be seen in Figure 1, the modulation of the electric field at frequencies $\omega_{f}<0$ gives rise to a response that is close to the maximum. In this case, the component $\phi_{k}$ of the field is dominant and much larger than all the other. Similar behavior can be observed for $\alpha<0$.

We now consider another aspect of non-normality, namely the transient response to a small initial perturbation of a stable lasing state in the absence of external forcing. Transient growth can occur if all the eigenvalues $\lambda_{k}$ of $L_{k}$ have $\Re\left(\lambda_{k}\right)<0$ and at least one eigenvalue of $L_{k}+L_{k}^{\dagger}$ is positive. We recall that the lasing solution becomes unstable and undergoes filamentation when at least one $\lambda_{k}$ such that $\Re\left(\lambda_{k}\right)>0$. From this simple condition we can easily find a physically very significant difference between positive and negative $\alpha$. The eigenvalues of $L_{k}+L_{k}^{\dagger}$ are invariant under change of sign of $\alpha$, and therefore, the value of the pump parameter $\rho$ where the largest eigenvalue of $L_{k}+L_{k}^{\dagger}$ becomes positive is the same for $\pm \alpha$. The situation is different for the eigenvalues $\lambda_{k}$ of $L_{k}$. The threshold value of $\rho$ for which there 


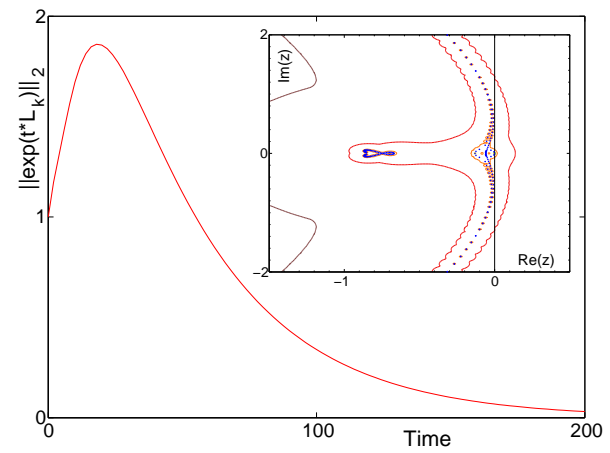

FIG. 3: Example of transient growth: plot of $\left\|\exp \left(L_{k} t\right)\right\|$ for $k=4$. Parameter values: $\alpha=2, \eta=0.09, \rho=0.6$. The insert shows the pseudo-spectrum of $L_{k}$ for $-20 \leq k \leq 20$. The contour lines correspond to $\left.\epsilon=10^{\{0,-1,-2,-3}\right\}$ from outside inward.

exists one eigenvalue $\lambda_{k}$ with $\Re\left(\lambda_{k}\right)>0$ is smaller for $\alpha$ positive than for $\alpha$ negative. This is consistent with the experimental observation that the filamentation threshold is higher in quantum dots than in bulk semiconductor lasers [18]. As a consequence, the range of control parameters where non-normal effects can be observed is larger for $\alpha<0$ than for $\alpha>0$.

A quantitative estimate of transient growth is given by (7) with $\boldsymbol{f}(x)=0$ : for each wave number $k$ of the perturbation, the maximum possible transient growth of the $k$-th Fourier component $\boldsymbol{v}_{k}$ is the norm $\left\|e^{t L_{k}}\right\|$. In order to estimate the maximum transient growth, it is very useful to introduce the $\epsilon$-pseudospectrum of $L_{k}$. This is defined as [15] $\Lambda_{\epsilon}(L)=\left\{z \in \mathbb{C}:\|R(z, L)\| \geq \epsilon^{-1}\right\}$. For $\epsilon=0$ this is just the standard spectrum; for $\epsilon>0$ the $\epsilon$ pseudospectra of $L_{k}$ are a family of strictly nested closed sets, which grow to fill the whole complex plane as $\epsilon \rightarrow$ $\infty$. The lower bound on maximal transient growth [10],

$$
\sup _{\Re(z)>0} \Re(z)\left\|\left(z-L_{k}\right)^{-1}\right\| \leq \sup _{t \geq 0}\left\|e^{t L_{k}}\right\|,
$$

can be interpreted geometrically in terms of the pseudospectrum: maximal transient growth occurs for wave numbers where the pseudo-spectrum extends as far as possible in the $\Re(z)>0$ half-plane for a given $\epsilon$. This is illustrated in Figure 3: the solution (4) is stable but the pseudo-spectrum extends sufficiently in the $\Re(z)>0$ half-plane for transient growth to take place. Note that this phenomenon is different from convective instabilities because the state considered here is stable and the group velocity of the perturbation may be null [21].

In conclusion, we have examined the effects of nonnormality in the context of a Swift-Hohenberg equation, a general model for spatio-temporal nonlinear processes. In general the linear stability operator of the solutions of this equation is non-normal. Therefore, we can expect that the main effects of non-normality, namely state dependent enhanced modulation response, excess noise and transient growth, can be seen in a variety of dynamical systems across many scientific disciplines. In the case used here as an example, a laser with asymmetric gain, the effects of non-normality are present even though the cavity modes are orthogonal. Moreover, the theory presented here provides tools to analyze and quantify the effects of non-normality: the resolvent allows to determine the effectiveness of enhanced modulation and transient growth, while the pseudo-spectrum provides a geometrical interpretation of these phenomena. For example, in lasers with asymmetric gain, they show that non-normal effects are more evident in lasers with negative $\alpha$ factor. Finally, this theory can be extended to determine the response to modulations of states with a complex time dependence [9]. This may be applied in synchronization experiments for secure optical communications [11], where modulations of solid-state lasers have been used to achieve controllable chaotic dynamics.

[1] G. H. C. New, J. Mod. Opt. 42, 799 (1995).

[2] K. Petermann, IEEE J. Quantum Electron. 15, 566 (1979).

[3] H. Haus and S. Kawakami, IEEE J. Quantum Electron. 21, 63 (1985).

[4] A. E. Siegman, Phys. Rev. A 39, 1253 (1989).

[5] M. V. Berry, J. Mod. Opt. 50, 63 (2003).

[6] W. J. Firth and A. M. Yao, Phys. Rev. Lett. 95, 073903 (2005).

[7] S. Longhi and P. Laporta, Phys. Rev. E 61, R989 (2000).

[8] B. F. Farrell and P. J. Ioannou, J. Atmos. Sci. 53, 2025 (1996).

[9] B. Farrell and P. Ioannou, J. Atm. Sci. 53, 2041 (1996).

[10] L. N. Trefethen, A. E. Trefethen, S. C. Reddy, and T. A. Driscoll, Science 261, 578 (1993).

[11] J. R. Terry and al., Phys. Rev. E 59, 4036 (1999). Y. Imai, H. Murakawa, and T. Imoto, Opt. Comm. 217, 415 (2003).

[12] J. Lega, J. V. Moloney, and A. C. Newell, Phys. Rev. Lett. 73, 2978 (1994).

[13] J. V. Moloney, J. Opt. B: Quantum Semiclass. Opt. 1, 183 (1999).

[14] J.-F. Mercier and J. V. Moloney, Phys. Rev. E 66, 036221 (2002).

[15] L. N. Trefethen, SIAM Review 39, 383 (1997).

[16] M. Embree and L. N. Trefethen, The pseudospectra gateway, URL http://web.comlab.ox.ac.uk/projects/ pseudospectra/.

[17] H. C. Schneider, W. W. Chow, and S. W. Koch, Phys. Rev. B 66, 041310 (pages 4) (2002).

[18] P. M. Smowton, E. J. Pearce, H. C. Schneider, W. W. Chow, and M. Hopkinson, Appl. Phys. Lett. 81, 3251 (2002).

[19] P. K. Jakobsen et al., Phys. Rev. A 49, 4189 (1994).

[20] M. V. Cross and P. C. Hohemberg, Rev. Mod. Phys. 65, 851 (1993).

[21] F. Papoff and R. Zambrini, Phys. Rev. Lett. 94, 243903 (2005). R. Zambrini and F. Papoff, Phys. Rev. Lett. 99, 063907 (2007). 